\title{
Rheological Modelling of Enzymatic Extrusion of Rice Starch
}

\author{
Rui. L. Tomás, ${ }^{a}$ Jorge. C. Oliveira ${ }^{a}{ }^{*} \&$ Kathryn. L. McCarthy ${ }^{b}$ \\ "Escola Superior de Biotecnologia, Universidade Católica Portuguesa, Rua Dr. António \\ Bernardino de Almeida, 4200 Porto, Portugal \\ ${ }^{\text {h}}$ Department of Food Science and Technology, University of California, Davis, CA 95616, \\ USA
}

\begin{abstract}
A rice starch mixture pre-mixed with thermostable $\alpha$-amylase was extruded in a pilot co-rotating twin-screw extruder, with the die replaced by a slit rheometer. The apparent viscosity at the slit wall was determined for a variety of operating conditions. The factors studied were barrel temperature $\left(70-110^{\circ} \mathrm{C}\right)$, water content $(550-650 \mathrm{~g} / \mathrm{kg}$ rice mixture $)$, flow rate $\left(8 \times 10^{-7}\right.$ to $\left.2.5 \times 10^{-6} \mathrm{~m}^{3} / \mathrm{s}\right)$ and enzyme concentration $(0.01-0.1 \mathrm{~g} / \mathrm{kg})$. The same screw configuration and rotational speed $(225 \mathrm{rpm})$ were used. A rheological model incorporating the integrated effect of extrusion and enzymatic activity was developed with a stepwise regression of the experimental data, analysing after each step the variability of the model parameters with the different factors. It was found that for the range of experimental conditions in question the influence of each factor could be considered separately with good results, leading to a power-law type model with a constant behaviour index. The parameters obtained describing the influence of water content, temperature and shear rate were within the range of available literature data for non-enzymatic extrusion and the influence of the enzyme concentration was well described by a power-law dependency.
\end{abstract}

\section{INTRODUCTION}

Extrusion has become an important processing technique in an increasing variety of foud processes (Harper, 1979).

*Author to whom correspondence should be addressed. 
The potential of an extrusion cooker to be operated as a bioreactor was only realised after it was observed, in the late $1970 \mathrm{~s}$, that some enzymes could remain active after extrusion cooking of cereal-based material under relatively mild processing conditions (Colonna et al., 1989). Until then, several authors had reported nearly quantitative inactivation of $\alpha$-amylases during extrusion cooking, under typical process conditions (Linko et al., 1983).

Given the many uses of modified starch, having well-defined characteristics, there is a significant potential in the development of techniques that can efficiently promote enzymatic modification of starch, with a good control of the extent of modification caused. This establishes the interest in mathematical descriptions of the enzymatic extrusion process as a basic tool for engineering design.

Efforts to simulate food extruders have drawn extensively from the polymer literature because of the general similarities. One of the major distinctions among the various models is the nature of the rheological assumptions concerning the extruding dough (Clark, 1978). Rheology provides an effective method for characterising changes in the material during extrusion cooking (MacKey and Ofoli, 1990). Rheological models can be applied to predict product quality and used in process control (Harper, 1981).

However, and although food extrusion is well established commercially, there is a small amount of published rheological data (Altomare et al., 1992). This fact is even more relevant when dealing with food doughs extruding in the presence of enzymes, where even the type of model to look for has not been analysed in the literature. Although it is intuitive that a time-dependent model such as the one proposed by Mackey and Ofoli (1990) could be applied, it is not known to what extent timedependency will affect the results.

Capillary rheometry has been used to determine the shear rheological properties of viscoelastic fluids. However, this procedure involves the use of several dies of different lengths and diameters. Moreover, it does not provide rheological data of melts that have experienced the completc processing history in the extruder (Senouci and Smith, 1988). Therefore, one is actually measuring the rheological properties of extrudates - that is, of food doughs that have already expanded and dehydrated. An alternative is the use of a slit die viscometer attached to the extruder, which performs an on-line measurement of the extruding material, prior to expansion and dehydration.

A laboratory-size twin-screw extruder coupled with a slit die viscometer offers a unique opportunity to add to the body of extruded food rheological information (Altomare et al., 1992). This type of rheometer is based on measurements of flow rate and pressure drop along the slit die, that are converted into estimates of the shear rate and shear stress at the wall following the well-established Rabinowitz procedure (Steffe, 1992).

Twin-screw extruders are normally starve-fed. This allows the adjustment of throughput and consequently shear rate without changing extruder conditions. The pressure gradient along the rectangular channel, necessary for the determination of the food melt viscosity, is directly measured using flush-mounted melt pressure transducers.

To analyse the rheological properties of the dough, several measurements are required at different experimental combinations of the various factors that are considered important in terms of the rheological model. 
The dependency of apparent viscosity on the measuring conditions in the slit rheometer is well described in viscometry (Steffe, 1992), following a stepwise buildup of the model. The model suggested in this work arises from assuming, empirically, that the rheometer/extruder combination will yield the same type of dependency. This means that the interpretation of results considers jointly the effects of the extruder conditions (i.e. the processing) and of the slit conditions, because only integrated results can be obtained experimentally.

The water contents used in this study were generally higher than those used in other extrusion modelling publications. The main reason for this is the fact that this study concerns the enzymatic extrusion of starch - that is, optimised for high moisture conditions (Linko, 1989).

The independent variables considered were moisture content and enzyme concentration in the feed stream, temperature of the extruding dough and flow rate.

\section{MATERIALS AND METHODS}

\section{Raw material}

The raw material used was a rice starch mixture containing $86 \%$ (minimum) starch with $1.5 \%$ protein content and $6.0 \%(\mathrm{w} / \mathrm{w})$ moisture content from California Natural Products (Lathrop, CA, USA). The starch powder was blended with tap water (hardness: $30 \mathrm{mg} / \mathrm{CaCO}_{3}$ ) in a Hobart mixer to the specified slurry water content. The enzyme used was Termamyl (NOVO, Novo Nordisk Enzymes Division, Bagsvaerd, Denmark), a liquid enzyme preparation containing a heat-stable $\alpha$-amylase expressed in and produced by a selected strain of Bacillus licheniformis. Linko (1989) reported a better enzymatic activity for starch added to the extruder as a premixed slurry. The slurry with the starch and the enzyme was fed to the extruder by a peristaltic pump.

\section{Extrusion}

Extrusion tests were performed with a System90 torque rheometer (Haake Buhler, Paramus, NJ, USA) that provides computer control and data acquisition for a MPC/ V-30 corotating twin-screw extruder (APV, Staffordshire, UK). The screws had four sections: a feed section with deep flights and $222 \mathrm{~mm}$ axial length, a kneading section with $33 \mathrm{~mm}$ axial length, a compression section with a decreased pitch and $98 \mathrm{~mm}$ axial length, and a metering section with very shallow flights and $28.5 \mathrm{~mm}$ axial length. This configuration was suitable for high moisture doughs. The extruder was operated in the starve-feeding mode, which means that the throughput was independent of the screw speed. The screws rotational speed was maintained at $225 \mathrm{rpm}$. The die was replaced by a slit rheometer.

Rheological determinations were based on data readings of the slit rheometer. Pressure along the $146 \mathrm{~mm}$ long slit rheometer was measured in three locations: $44.45 \mathrm{~mm}, 82.55 \mathrm{~mm}$ and $120.65 \mathrm{~mm}$ from the entrance. The pressure transducers (Dynisco, type PT422A) were calibrated at each operating temperature for accurate readings. To increase accuracy, three different pressure ranges were selected in the transducers: $0-34$ atm near the die exit, $0-68$ atm in the middle and $0-102 \mathrm{~atm}$ near the extruder barrel. 
TABLE 1

Range of Operating Conditions

\begin{tabular}{llll}
\hline $\begin{array}{l}\text { Flow rate } \\
\left(\mathrm{m}^{3} / \mathrm{s}\right)\end{array}$ & $\begin{array}{l}\text { Added } \\
\text { water } \\
\text { content }(\%)\end{array}$ & $\begin{array}{l}\text { Temperature } \\
\left({ }^{\circ} \mathrm{C}\right)\end{array}$ & $\begin{array}{l}\text { Enzyme } \\
\text { concentration } \\
\text { (g enzyme per } \\
\text { I00 g rice } \\
\text { mixture })\end{array}$ \\
\hline $\begin{array}{l}8.3 \times 10^{-7} \text { to } \\
2.5 \times 10^{-6}(\text { steps }\end{array}$ & $55,60,65$ & $70,90,100,110$ & $0.01,0.02,0.03,0.04$, \\
of $\left.0.17 \times 10^{-6}\right)$ & & & $0.05,0.07,0.10$ \\
\hline
\end{tabular}

A computerised data acquisition system (Rheocord System 90, Haake Buhler) attached to the rhcometer was used to record the tcmpcratures (mclt and set temperatures), the three pressure readings, the screw speed and the torque, in steps of $6 \mathrm{~s}$. Data collection was initiated after steady-state was reached, as determined by constant pressure and torque readings.

Added moisture, amount of enzyme added, barrel temperature and flow rate were the conditions varied in this study. The operating conditions used were all the combinations of the values detailed in Table 1 with some replicates, in a total of 573 points.

The temperature in the slit rheometer was always maintained equal to that in the barrel. Product temperatures in different zones of the barrel and in the slit die were set using the control software. Temperatures and deviations from the set temperatures were measured by eight thermocouples along the extruder barrel and two in the slit die torque rheometer and controlled with electrical heating and air cooling systems, in a range of $\pm 2^{\circ} \mathrm{C}$.

The selected amounts of enzyme added were based on the dosage normally used in the paper industry (Lovin and Wheeler, 1990).

\section{Slit viscometry}

The following assumptions, generally assumed in slit viscometry (Altomare et al., 1992) were used to interpret the slit data:

(1) Fully developed flow

(2) Isothermal conditions

(3) Negligible viscous heat dissipation

(4) No slip at the walls

(5) Laminar flow regime

(6) Negligible end effects

(7) No chemical or physical reactions, interactions or changes in state along the rheometer

(8) Shear history in the rheometer can be ignored.

The apparent viscosity at the slit wall was calculated from the pressure drop and flow rate in the slit, using the flow equations. It can be demonstrated easily (Steffe, 1992) that, for the slit geometry, neglecting end effects and assuming the no-slip condition at the wall, the shear stress at the wall $\left(\tau_{w}\right)$ is given by: 


$$
\tau_{\mathrm{w}}=\frac{\Delta P \times \mathrm{H}}{2 \mathrm{~L}}
$$

where $\Delta P$ is the pressure drop along the slit and $L$ and $H$ are the slit length and height, respectively. The shear stress at the wall $\left(\gamma_{w}\right)$ is given by:

$$
\gamma_{w}=\Gamma\left(\frac{2 n^{\prime}+1}{3 n^{\prime}}\right)
$$

where $n^{\prime}$ is the slope of the tangent of the logarithmic plot of $\tau_{w}$ versus $\Gamma$; that is:

$$
n^{\prime}=\frac{\mathrm{d}\left(\ln \tau_{\mathrm{w}}\right)}{\mathrm{d}(\ln \Gamma)}
$$

with $\Gamma$ being the shear rate if the fluid would be Newtonian:

$$
\Gamma=\frac{6 Q}{X \times \mathrm{H}^{2}}
$$

with $X$ being the slit width and $Q$ the volumetric flow rate.To obtain $n^{\prime}$, it is necessary to determine several values of shear stress and shear rate by varying the flow rate. However, this affects the operating conditions because the slit is connected to the extruder. It would be necessary to vary the flow rate passing through the slit without affecting the flow rate through the extruder to obtain the correct value of $n^{\prime}$. Since this is not possible, it must be assumed that the flow rate does not affect $n^{\prime}$ significantly. This hypothesis is preferable over the alternative of assuming $\gamma_{\mathrm{w}}=\Gamma$, which implics $\mathbf{n}^{\prime}=1$.

The apparent viscosity is the ratio between the shear stress and the shear rate. This analysis does not imply any type of rheological behaviour of the fluid. If a power law can be applied, then $n^{\prime}$ is constant and equal to the fluid behaviour index $n$.

Since three measuring locations existed, it could be verified experimentally that the end effects were negligible, which is reasonable for the high $L / H$ ratio of the slit. The use of a slit rheometer has the disadvantage that results are obtained for a range of high shear rates only and if the fluid is non-Newtonian, as expected, this is not a full characterisation of the fluid rheological behaviour. Each value was the result of continuous readings for a minimum of $1 \mathrm{~min}$.

\section{Rheological model}

The methodology selected for determining the different parameters of the rheological model was a stepwise regression.

Many rheological models have been proposed for non-Newtonian fluids. However, it has been reported that most often the simple power law was adequate for many foods (Clark, 1978) for a relatively small range of shear rates:

$$
\eta=K \gamma^{n-1}
$$


The variation of the behaviour index $(n)$ and the consistency index $(K)$ with the operating variables was analysed and the model variables incorporated one by one. This procedure was preferred to the establishment of an empirical model and therefore develops as the results are analysed, which implies that no other assumption is made that is not justified by the experimental data.

\section{RESULTS AND DISCUSSION}

Linear regressions with eqn (5) were performed for the several sets of data, grouped by constant temperature, moisture and enzyme concentrations ( 84 sets, as can be seen in Table 1). No particular trend was found for any of the factors studied (temperature, enzyme concentration and water content), while a normal distribution of the residuals of the individual values of $n$ around the average, 0.35 was observed, with a standard deviation of 0.03 . It is important to note that the parameters of the power-law model are highly collinear. This means that for a value of $n$ that is within the confidence limits there is a small range of $K$ values that form a pair of parameters whose predictions are not statistically different from the solution. However, when analysing only one parameter, a large spread can be found. If a constant value of $n$ appears to describe all the results well, it is essential to recalculate the best values of $K$ for that $n$ and disregard the previous values.

If $n$ can be considered constant, the recalculated consistency indexes will then be divided in the several contributing factors in the same way. In relation to temperature, Morgan et al. (1989) have assumed a constant behaviour index and an exponential dependency of the consistent index with temperature following an Arrhenius type equation.

$$
K=K_{\mathrm{T}} \mathrm{e}^{-\frac{\Delta E_{\mathrm{r}}}{R}}\left(\frac{1}{T}-\frac{1}{T_{\mathrm{r}}}\right)
$$

where $\Delta E_{\mathrm{r}}$ is the activation encrgy for viscous flow processes, $R$ is the gas constant and $T_{\mathrm{r}}$ a reference temperature. The use of a reference temperature decreases the collinearity of the confidence region and is therefore preferable for the regressions. The lowest temperature, $70^{\circ} \mathrm{C}$, was considered. Several sets of data can therefore be analysed, determining the several $K_{\mathrm{T}}$ and $\Delta E_{\mathrm{r}}(21$ sets, as can be seen in Table 1). The activation energy varied slightly with the remaining variables (enzyme concentration and moisture content), but within the range of experimental error; therefore, for modelling purposes, an average was used $\left(\Delta E_{\mathrm{r}} / R=4077 \pm 271\right)$, and values of $K_{\mathrm{T}}$ for this fixed activation energy were obtained. The Arrhenius parameters, like the power-law parameters, are also highly collinear, so the values of $K_{\mathrm{T}}$ that fit the results best for a fitted value of $\Delta E_{\mathrm{r}} / R$ must also be recalculated.

The following step was to incorporate the effect of the enzyme concentration in the rheological model. The incorporation of the concentration of different compounds in rheological models has been reported in literature as following a power-law model (MacKey and Ofoli, 1990), but few have involved enzymes. Taking this suggestion anyway:

$$
K_{\mathrm{T}}=K_{\mathrm{E}}[E]^{\prime \prime}
$$


Regressions of the several sets of data were done for the different moisture levels used in the study, and fit to eqn (7) (three sets, as can be seen in Table 1). The regressions obtained were quite good, and the power index remained fairly constant for the different moisture contents studied $(a=-0 \cdot 457 \pm 0 \cdot 05)$. Once again, the best values of $K_{\mathrm{E}}$ for a fixed value of $a(-0.457)$ were recalculated.

The effect of moisture content was then incorporated in the changes observed in the consistency indexes $\left(K_{\mathrm{E}}\right)$. Clark (1978) has used an exponential form of variation of viscosity with moisture with some success, and therefore this kind of dependence was selected:

$$
K_{\mathrm{E}}=K_{\mathrm{M}} \mathrm{e}^{h\left(M C-M C_{\mathrm{r}}\right)}
$$

With $M C_{\mathrm{r}}$ being a reference moisture content $(0 \cdot 55)$. In fact, the exponential relationship between viscosity and water content is well documented in the literature (Altomare et al., 1992), although not for the specific case of enzymatic extrusion. A

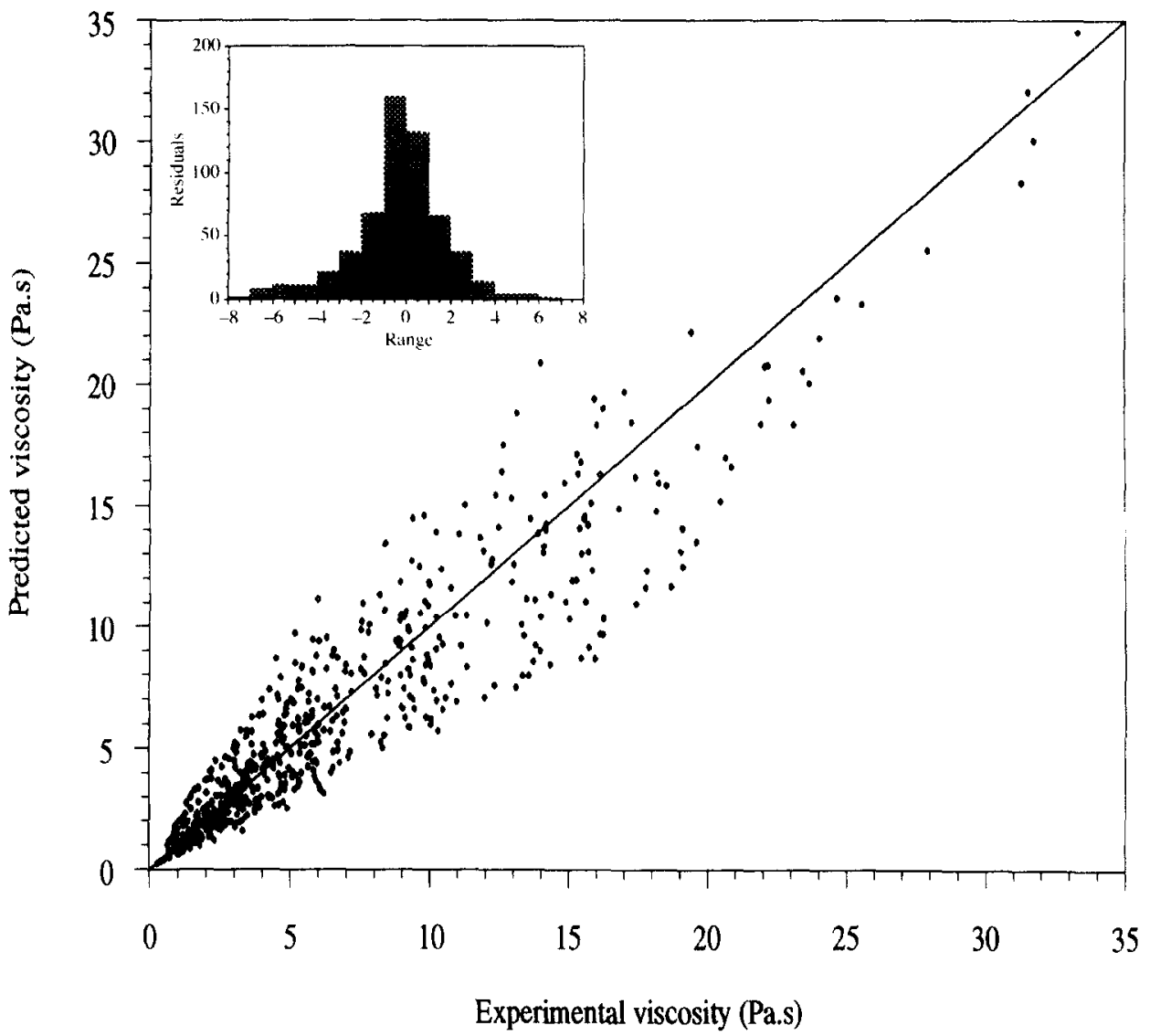

Fig. 1. Diagnosis plot of the correlation between experimental data and model predictions. (The small plot shows the frequency distribution of the residuals.) 


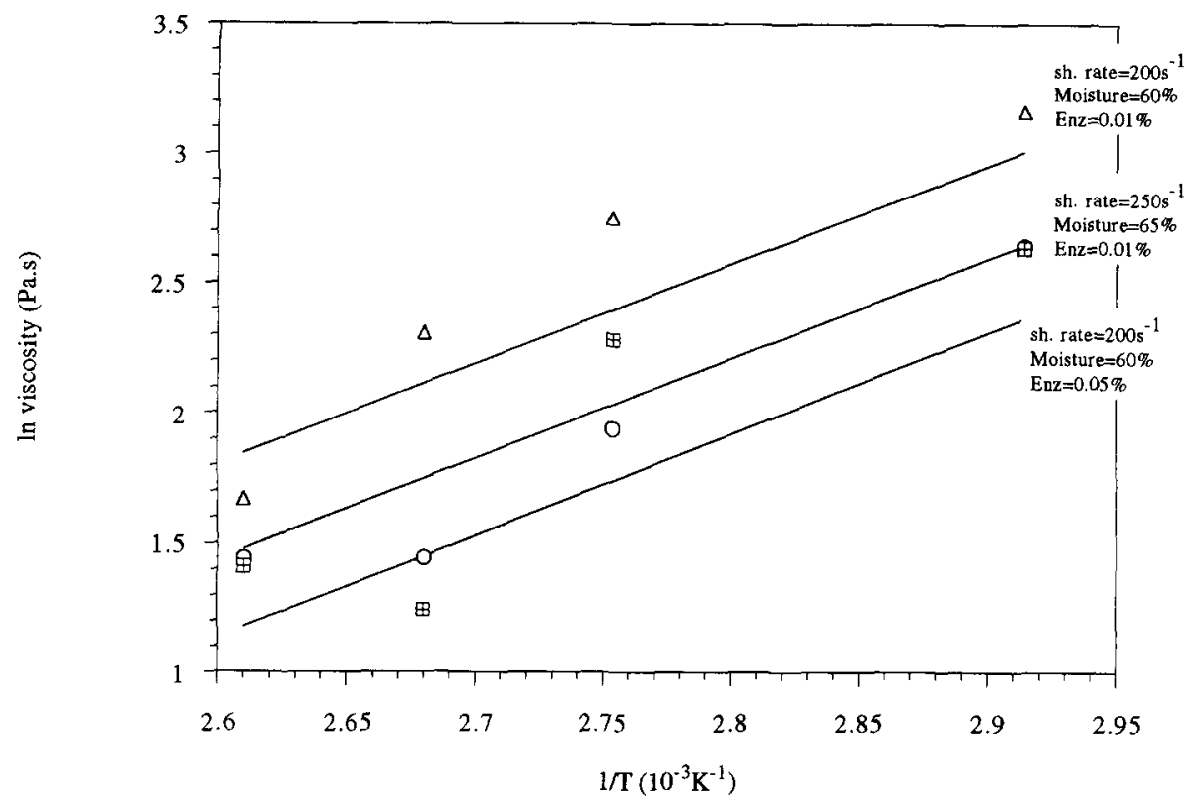

Fig. 2. Influence of temperature on viscosity for typical sets of data. Full lines show the corresponding model predictions.

reference moisture content of $55 \%$ was considered. The parameters obtained were finally $b=-4 \cdot 54 \pm 3 \cdot 22$ and $K_{\mathrm{M}}=102 \cdot 8 \pm 1 \cdot 3$.

Grouping eqns (5)-(8) leads to the overall model:

$$
\eta=102 \cdot 8 \mathrm{e}^{-4077}\left(\frac{1}{T}-\frac{1}{343 \cdot 15}\right)[E]^{-0.46} \mathrm{e}^{-454(M C-0.55)} \gamma^{0.35}
$$

The parameters obtained with this stepwise regression were compared with some published values. However, because little work has been done combining rheological modelling of enzymatic wet extrusion, it was not possible to compare the same range of conditions, especially for the parameter that describes the effect of the enzyme concentration. For a moisture content of $85 \%$ and a temperature of $54^{\circ} \mathrm{C}$, Luxenburg et al. (1985) reported a behaviour index of $n=0 \cdot 37$. Jao et al. (1978) extruded defatted soya in a range of temperatures from 15 to $160^{\circ} \mathrm{C}$ and a moisture content of 25-63\%, and obtained a variation of $n$ between $0 \cdot 16$ and $0 \cdot 75$. Cervone and Harper (1978) studied the extrusion of pregelatinised corn flour using a range of temperatures between 90 and $150^{\circ} \mathrm{C}$ and a range of moistures between 22 and $35 \%$, and obtained $n=0.36$ and an activation energy $\Delta E_{\mathrm{r}} / R=4390$, which are fairly close to the parameters obtained in this study. They included also the effect of moisture content in the rheological behaviour of food extrudates and obtained a $b$ value of $-6 \cdot 8$, again quite close to the one obtained in this study. However, their results were obtained by off-line rheological measurements. 
Figure 1 shows a diagnosis plot of the experimental data compared to eqn (90 predictions. The residuals between predicted and experimental values are also shown in Fig. 1, where it can be seen that a close to normal distribution, with average zero, was obtained. The model is therefore statistically acceptable, not showing any particular trend or systematic error.

Figures 2-4 show some representative results, where the adequacy of the model to predict the effect of individual factors is shown. The experimental data are compared with the predictions of eqn (9), not with the best individual regressions of eqn (6), eqn (7) or eqn (8). The axes are such that model predictions are straight lines in the plots shown.

\section{CONCLUSIONS}

A stepwise model starting from a power-law equation and combining the effect of several variables in the viscosity of extruding rice flour in the presence of $\alpha$-amylase was obtained. The stepwise analysis showed that in the range of operating variables used the effects of each variable could be considered independently, within the

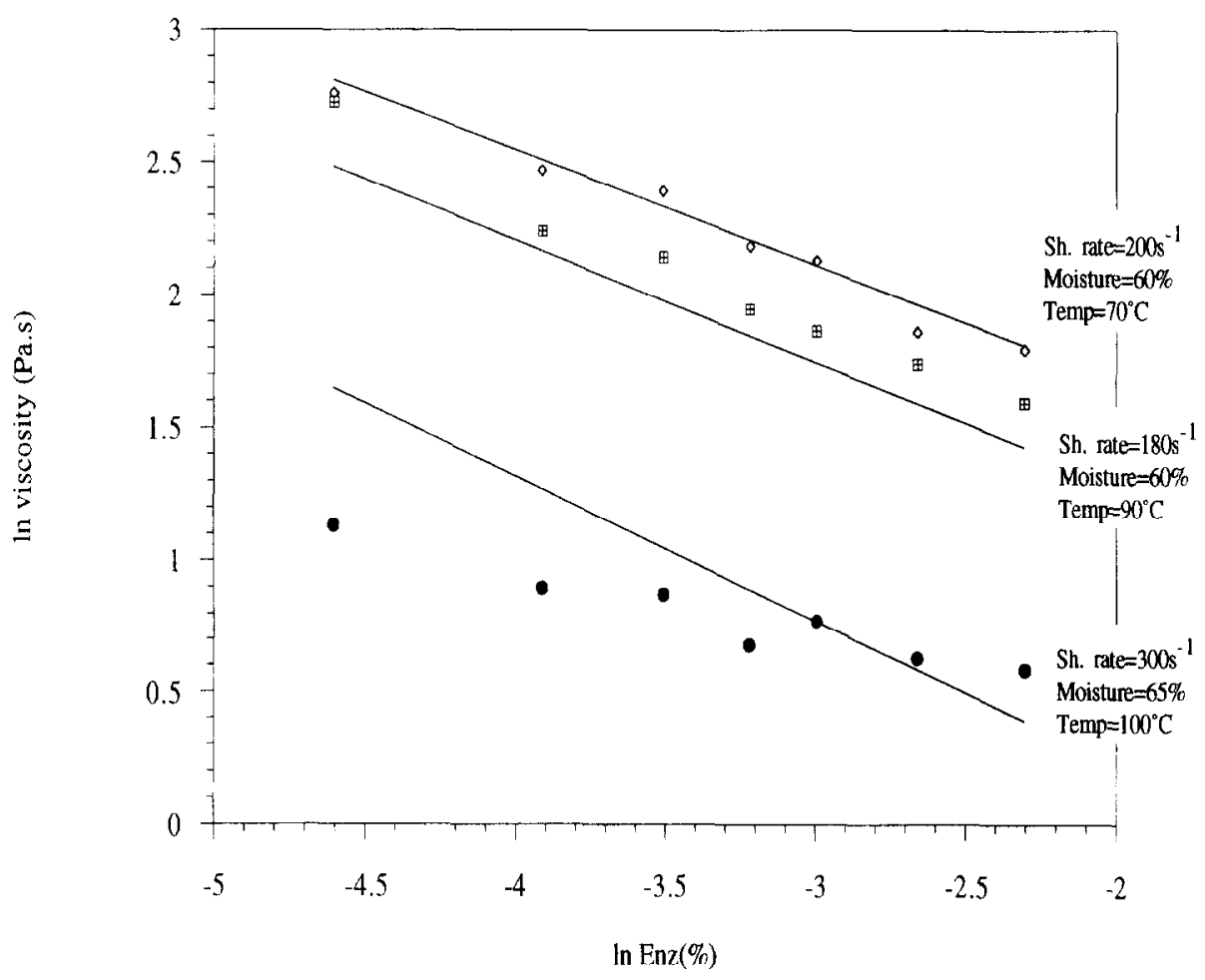

Fig. 3. Influence of enzyme concentration on viscosity for typical sets of data. Full lines show the corresponding model predictions. 


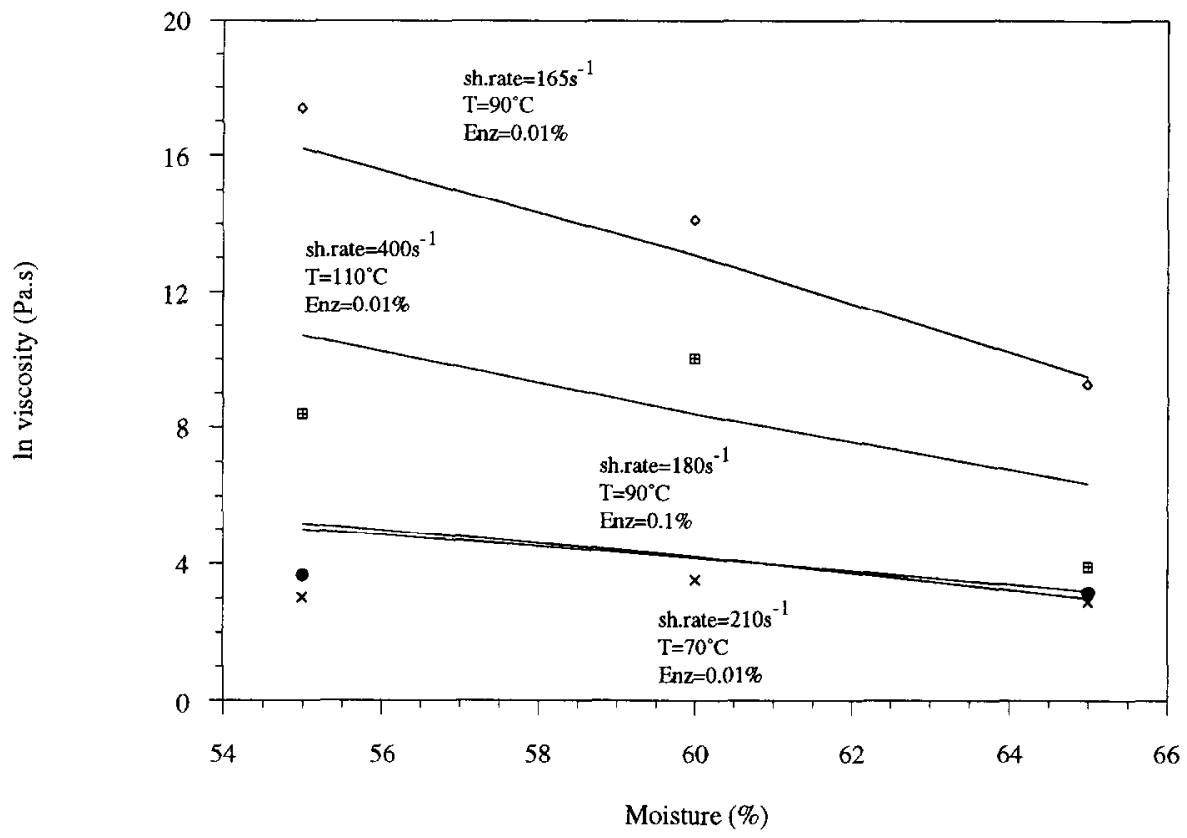

Fig. 4. Influence of moisture content on viscosity for typical sets of data. Full lines show the corresponding model predictions.

accuracy of the experimental error. Results showed a good fit of the experimental data with an overall model.

The behaviour index was approximately constant within the range of variables used. Similarly, constant exponential parameters were found for the influence of temperature and added water in the consistency index, together with a power-law dependency on the enzymatic concentration.

\section{ACKNOWLEDGEMENTS}

R.L.T. and J.C.O. gratefully acknowledge support by the European Commission (AAIR programme, project 'Industrial non-food uses of thermoplastic starch'). R.L.T. acknowledges financial support from Junta Nacional de Investigaçcão Científica e Tecnológica.

\section{REFERENCES}

Altomare, R. E., Anelich, M. \& Rakos, R. (1992) An experimental investigation of the rheology of rice flour dough with an cxtruder-coupled slit die rheometer. In Food Extrusion Science and Technology, ed. J. L. Kokini, C. Ho \& M. V. Karwe, pp. 233-254. Marcel Dekker, New York. 
Cervone, N. W. \& Harper, J. M. (1978). Viscosity of an intermediate moisture dough. J. Food Process Eng., 2, 974-977.

Clark, J. P. (1978). Dough rheology in extrusion cooking. Food Technol., 7, 73-82.

Colonna, P., Tayeb, J. and Mercier, C. (1989) Extrusion cooking of starch and starchy products. In Extrusion Cooking, ed. C. Mercier, P. Linko and J. M. Harper, pp. 247-319. American Association of Cereal Chemists, St. Paul, MN.

Harper, J. M. (1979). Food extrusion. CRC Crit. Rev. Food Sci. Nutr., 11, 155-215.

Harper, J. M. (1981) Extrusion of Foods, Vol. 1. CRC Press, Boca Raton, FL.

Jao, Y. C., Chen, A. H., Leandowski, D. \& Irwin, W. E. (1978). Engineering analysis of soy dough. J. Food Process Eng., 2, 97-112.

Linko, P. (1989) Extrusion cooking in bioconversions. In Extrusion Cooking, ed. C. Mercier. P. Linko and J. M. Harper, pp. 235-245. American Association of Cereal Chemists, St. Paul, MN.

Linko, P., Linko, Y.-Y. \& Olkku, J. (1983). Extrusion cooking and bioconversions. J. Food Eng., 2, 243-257.

Lovin, J. C. and Wheeler, H. R. (1990) Enzyme conversion of starch for paper coating. In Starch and Starch Products in Paper Coating, ed. R. L. Kearey and H. W. Mercier, pp. 46-60. Tappi Press, Atlanta, GA.

Luxenburg, L. A., Baird, D. G. \& Joseph, E. G. (1985). Background studies in the modeling of extrusion cooking processes for soy flour doughs. Biotechnol. Progress, 1, 33-38.

MacKey, K. L. \& Ofoli, R. Y. (1990). Rheological modeling of corn starch doughs at low to intermediate moisture. J. Food Sci. 55, 417-423.

Morgan, R. G., Steffe, J. F. \& Ofoli, R. Y. (1989). A generalized viscosity model for extrusion of protein doughs. J. Food Process Eng., 11, 55-78.

Senouci, A. \& Smith, A. C. (1988). An experimental study of food melt rheology. I. Shear viscosity using a slit die viscometer and a capillary rheometer. Rheol. Acta, 27, 546-554.

Steffe, J. F. (1992) Slit viscometry. In Rheological Methods in Food Process Engineering, ed. J. F. Steffe, pp. 75-77. Freeman Press, East Lansing, MI. 